\title{
About the need for optical gear measurements using a beam diversion mechanism in order to obtain truthful surface texture parameters
}

\author{
Beurteilung der Notwendigkeit einer Strahlumlenkung für optische Zahnradmessungen zur \\ Bestimmung korrekter Oberflächentexturkennwerte
}

DOI 10.1515/teme-2019-0044

Zusammenfassung: In dem folgenden Artikel konnte anhand verschiedener Messreihen gezeigt werden, dass die optische Erfassung von Bauteiloberflächen unter verschiedenen Betrachtungswinklen auch zu unterschiedlichen Oberflächentexturkennwerten führt. Die Ergebnisse rechtfertigen die Entwicklung einer daraufhin angepassten messtechnischen Erfassung von Zahnradflanken mit Hilfe einer optischen Strahlumlenkung.

Schlüsselwörter: Oberflächentexturkennwerte, Fokusvariation, Neigungswinkel, Zahnradmessung.

\begin{abstract}
The following article shows, that the optical characterisation of work piece surfaces with varying angles of inclination leads to different surface texture parameters. These results justify the development of a customised measurement setup using a optical beam diversion mechanism for the correct evaluation of gear wheel flanks.
\end{abstract}

Keywords: Surface texture parameters, focus variation, angle of incidence, gear wheel measurement.

\section{Introduction}

The intended function of a gear stage is dependent on the geometrical correctness of both tooth flanks relative to each other during contact in the state of operation. Different causes for geometrical deviations are imperfections of the manufacturing processes, deviations induced by the assembly or wear and tear impacts. The former and the latter effect are detected using dimensional metrology tools

\footnotetext{
*Corresponding author: Andreas Michael Müller, Lehrstuhl für Fertigungsmesstechnik (FMT), Friedrich-Alexander-Universität Erlangen-Nürnberg (FMT), Nägelsbachstraße 25, 91052 Erlangen, E-Mail: andreas.mueller@fmt.fau.de

Tino Hausotte, Lehrstuhl für Fertigungsmesstechnik (FMT), Friedrich-Alexander-Universität Erlangen-Nürnberg (FAU), Nägelsbachstraße 25, 91052 Erlangen, E-Mail: tino.hausotte@fmt.fau.de
}

such as tactile coordinate measurement machines, various optical systems or industrial X-ray computed tomography. Traditional tactile gear wheel inspection typically only consists of one profile and one lead line on both flanks of four teeth plus one point at the center of all flanks [3]. While these measurements cannot detect any twist deviations, they also cannot create an areal illustration of the contacting gear flank sectors and thus are insufficient for the complete determination of the potential wear volume. Additionally, tactile measurements are mostly unsuitable of measuring surface textures due to the morphological filtering of the probing sphere. In contrast, optical measurement systems can offer the possibility to measure those target quantities correctly with a high resolution, if a high incidence angle of the optical axis towards the target surface can be set up. ISO 25178: Geometric Product Specifications (GPS) - Surface texture: areal is an ISO collection of international standards relating to the analysis of 3D areal surface texture [8]. Part 6 of ISO 25178 classifies methods for measuring surface texture, including contact and non-contact topographical and profilometric instruments as well as instruments functioning by integration [4]. The ISO 1328-1 defines the deviations relevant to flanks of gear teeth [6]. The profile evaluation range of a gear tooth is in contact with a second gear wheel during the state of operation, thus the texture properties of both flanks are influencing the amount of wear and tear. The determination of the gear tooth surface texture using optical topographical instruments is affected by the optical approachability. Depending on the gear module, an on average perpendicular alignment of the optical axis towards the profile evaluation range of a gear tooth is not possible due to the shading of the neighbouring gear tooth. The straight-toothed gear wheels targeted for examination exhibit a module $m$ of $1 \mathrm{~mm}$. Consequently, in the case of non-destructive examination the gear tooth surface can only be measured optically with an angle of incidence significantly smaller than $90^{\circ}$ (angle of inclination of the 
measurement object greater than $0^{\circ}$ ). This paper aims to contribute to the discussion of the determination of surface texture parameters in the context of optical gear measurements utilizing the commercial measurement system Alicona Infinite Focus G4 [1] (measurement principle: focus variation) by demonstration of the necessity of a preferably perpendicular acquisition of the gear flank surface. In this work we could show, that the observed surface texture parameters change substantially dependent on the angle of inclination of the measurement surface, which laid the groundwork for the future development of a beam diversion mechanism for accurate gear measurements.

\section{Methodology}

The foundation of the subsequent examination was set by the construction and manufacturing of a milled test specimen (material X5CrNi18-10), as shown in Fig. 1. A flattened area (right side) contains the rotation axis of the cylinder and is provided with nine reference markers. The area marked with the blue square (right side) defines the measuring range for the following examinations.

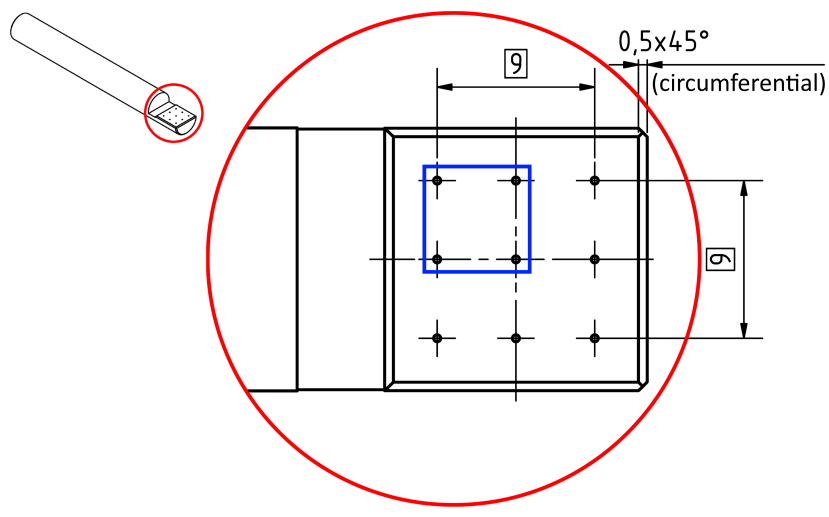

Fig. 1: Test specimen with diameter of $15 \mathrm{~mm}$. The range inside the blue patch (right) was used for all measurement series.

The work piece was then mounted onto a rotatory stage and several measurement series (each consisted of 20 measurement repetitions) featuring different inclination angles towards the xy plane of the measurement system ( $\mathrm{z}$ defining the optical axis) were carried out. The measurement system consists of the Alicona Infinite Focus G4 (measurement principle: focus variation) with an objective featuring $5 \mathrm{x}$ magnification, a working distance of $23.5 \mathrm{~mm}$ and a numerical aperture of 0.15 . This results in a (half) angle of acceptance of approx. $8.63^{\circ}$. For this lens the manufacturer gives the following specifications [1]: vertical resolution $410 \mathrm{~nm}$, lowest measurable roughness
Sa $0.375 \mu \mathrm{m}$. The lighting was adjusted automatically by the measurement system. The measurement series were then evaluated using two different methods: The first evaluation method is characterised by the determination of the surface height parameters defined in ISO 25178 using MountainsMap Premium 7.4.8803 [2]. For each sin-

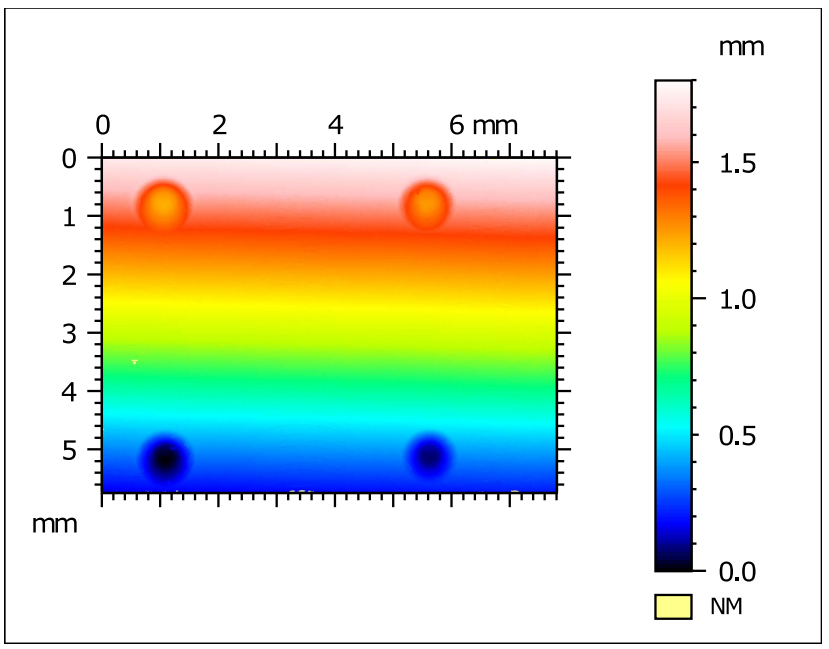

Fig. 2: Exemplary measurement (inclination angle $15^{\circ}$ ).

gle measurement of a measurement series (Fig. 2), exactly the same centre area inside the blue patch without the reference markers was selected and analysed. The flat evaluation range was oriented such that the surface normal vector was parallel aligned with the vector $[\mathrm{X}$ Y Z $]=\left[\begin{array}{lll}0 & 0 & 1\end{array}\right]$ to enable a correct determination of the surface height parameters (see ISO 25178-2:2012-09 section 3.1.2 [5]). After that, the S-L-surface was determined using a robust Gaussian filter following ISO 16610-71 [7] with a Nesting index of $0.8 \mathrm{~mm}$. After this pre-processing (Fig. 3), the areal surface height parameters were determined. These are described in detail in ISO 25178-2 and include the following quantities: Sq ('root mean square height of the surface', equivalent to the standard deviation of heights), Ssk ('skewness of the height distribution'), Sku ('kurtosis of the height distribution', measure of the sharpness of the roughness profile), Sp ('maximum height of peaks'), Sv ('maximum height of valleys'), Sz ('maximum height of the surface') and Sa ('arithmetical mean height of the surface', used generally to evaluate surface roughness). The second method of evaluation is described by the framework of the single point uncertainty (SPU), which was used successfully for various usage scenarios in dimensional metrology [9-12]. Originating from homogeneously distributed surface points on a reference surface (also called sampling points $\mathrm{SP}_{\mathrm{k}}$ ), the distances to each single measu- 


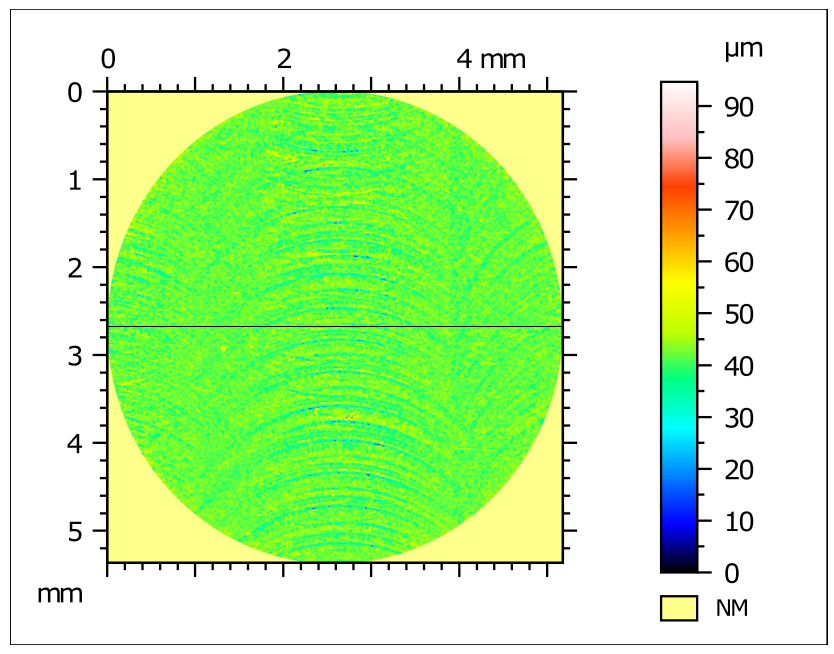

Fig. 3: Exemplary measurement (inclination angle $15^{\circ}$ ) after pre-processing using MountainsMap.

red surface of a measurement series are calculated. Here, the sampling strategy normal vector was used (Fig. 4). That means, that the signed distances are calculated in

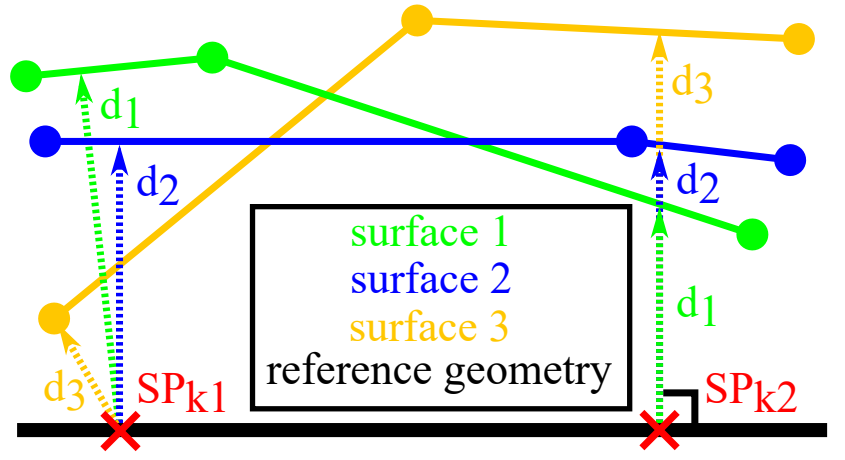

Fig. 4: Principle of the SPU with sampling strategy nearest neighbour (left, not used) and normal vector (right).

the direction of the surface normal vector. Consequently, each $\mathrm{SP}_{\mathrm{k}}$ is associated with $n$ calculated distances (one for each measurement repetition). The method then enables in principle the evaluation of the areal distribution of the systematic measurement error (represented by the mean distances associated with each sampling point) as well as the random measurement error (represented by the standard deviation of the distances associated with each sampling point, which is also called single point scatter $(S P C))$. The combination of both errors results in the single point (measurement) uncertainty. For this evaluation, a reference measurement of the measurement object is necessary. In case only the nominal geometry of the measurement object is available, the geometric deviations of the work piece and the systematic measurement error can not be separated, but the SPC could be evaluated nonetheless. In this contribution, only the SPC is evaluated, but neither the nominal geometry nor a reference geometry were known for these measurements. Instead, the sampling start surface (consisting of all $\mathrm{SP}_{\mathrm{k}}$ )) for each measurement series was defined by one plane fit (used points: $5 \sigma$ ) of all measured surfaces of that measurement series using GOM Inspect. In case of a perpendicular measurement of a flat area, the surface normal vector and the optical axis are aligned in parallel. In order to examine the effect of an inclined optical measurement, the SPC was additionally calculated in the direction of the optical axis, which leads to identical sampling directions in case of a perpendicular measurement, but different directions depending on the optical inclination angle between the measurement object and the optical axis of the measurement system during the measurement. It is expected that both sampling methods produce different results, thus showing that the measurement result (and therefore also the measurement uncertainty) changes for different angles of inclination.

\section{Results}

The figures shown on the next page prove the influence of the angle of inclination of the test specimen with respect to the optical axis of the measurement system onto the observed surface texture parameters (Fig. 5). The observed $\mathrm{Sa}$ and $\mathrm{Sq}$ values are both reduced significantly as soon as the angle of inclination is larger than the angle of acceptance of the lens $\left(8.63^{\circ}\right)$. Additionally, both parameters are very stable with very low associated error bars. The parameters Sv, Sz and Sp are also reduced for higher angles of inclination compared to a perpendicular measurement, but the scatter of those values is larger compared to the Sa and Sq values, mainly because these parameters are determined by single (outlier) measurement points. The determined Sa values are larger than the minimum measurable roughness value defined for the used lens by the manufacturer, thus confirming the suitability of the used measurement set up for these examinations.

As expected, the differences between the observed single point scatter values for different sampling vectors clearly increase with an increasing angle of inclination. (Fig. 6). Each colour represents a different inclination angle of the measurement object. The dashed line stands for the sampling vector in the direction of the surface normal vector and the solid line for the sampling in the direction of the optical axis, respectively. 


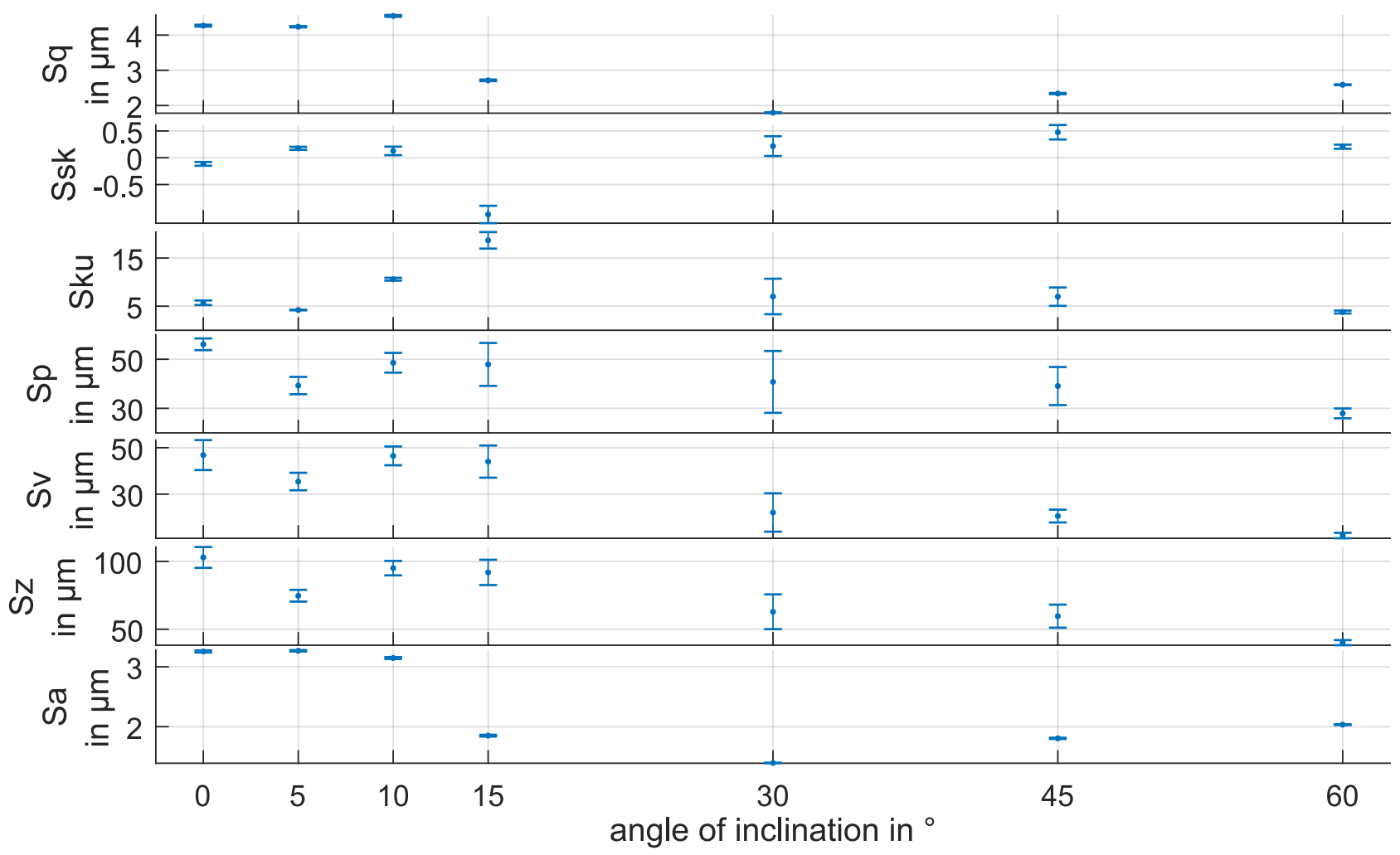

Fig. 5: Visualising the effects of different angles of inclination of the measurement object onto the measured surface texture parameters. Each data point represents the mean value of 20 repeated measurements, error bars represent three standard deviations.

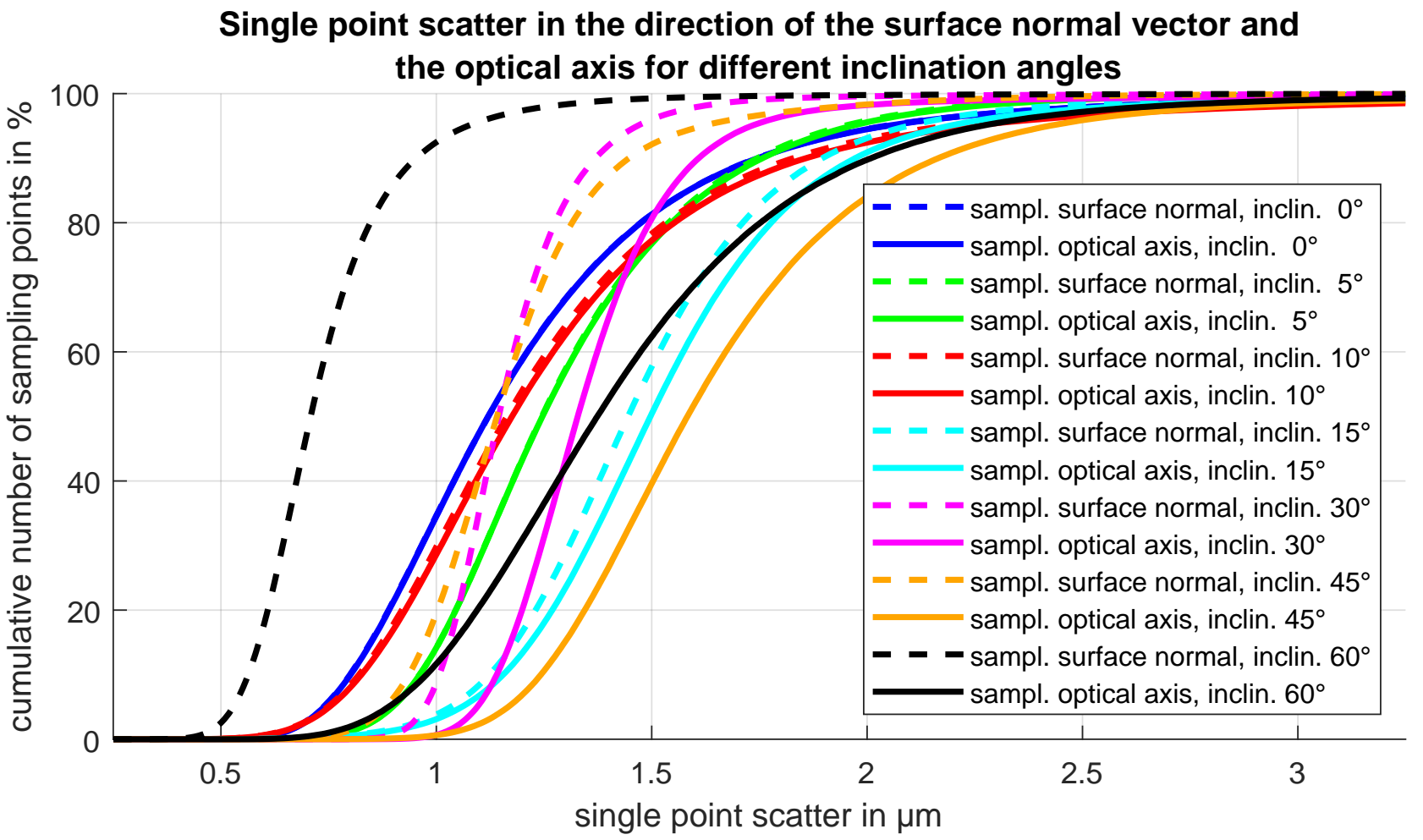

Fig. 6: Single point scatter for different inclination angles and sampling directions: dashed lines show results for sampling using the surface normal vector, solid lines correspond to sampling in the direction of the optical axis. 
For low inclination angles $\left(0^{\circ}, 5^{\circ}\right.$ and $\left.10^{\circ}\right)$ the results for both sampling directions are almost identical, thus the dashed and the solid line mostly lie on top of each other. For all measurement series, the sampling in the direction of the surface normal vector produces equal or smaller single point scatter values compared to the sampling in the direction of the optical axis.

\section{Discussion and Summary}

The evaluation of overall 140 single measurements $(7 \times 20$ measurement repetitions) showed, that angles of inclination of larger than $10^{\circ}$ to $15^{\circ}$ lead to different observations, compared to rather vertical measurements. The angle of acceptance of the used lens was just below $10^{\circ}$, which certainly impacted the results for higher angles of inclination. These results confirm the necessity of perpendicular optical surface measurements, if the texture parameters are part of the measuring task. Consequently, gear measurements with an angled observation are expected to produce faulty surface texture parameters. This can substantially influence tribological examinations. The observed effects could become much larger, if parts using different manufacturing techniques than milling, as used in this demonstration. Therefore, these results lay the groundwork for future work in order to modify the described measurement set up for gear wheels, resulting in steeper optical observation angles and thus ultimately in measurements with lower measurement uncertainties.

Revisiting the observations shown in Fig. 6, the median scatter values $\left(50^{\text {th }}\right.$ percentile) do not strictly increase with increasing inclination angles in case of the sampling in the direction of the optical axis or decrease in case of the sampling in the direction of the surface normal vector, respectively. It is possible that the automatic adjustment of the lighting settings of the measurement device as well as the characteristics of the surface of the examined measurement object (see milling tracks in Fig. 3) caused this.

Acknowledgements: The authors would like to thank the German Research Foundation (DFG) for supporting the research project FOR 2271 process-oriented tolerance management based on virtual computer-aided engineering tools under grant number HA 5915/9-1.

\section{Literatur}

[1] Alicona Imaging $\mathrm{GmbH}$. Dimensional metrology \& roughness measurement: Infinitefocus, 2019. URL https://www.alicona. com/en/products/infinitefocus/.

[2] Digital Surf SARL. Mountainsmapß premium: Surface imaging, analysis \& metrology software par excellence, 19962013. URL https://www.digitalsurf.com/wp-content/ uploads/2018/06/MountainsMap_Premium.pdf.

[3] G. Goch, K. Ni, Y. Peng, and A. Guenther. Future gear metrology based on areal measurements and improved holistic evaluations. CIRP Annals, 66(1):469-474, 2017. ISSN 00078506. 10.1016/j.cirp.2017.04.046.

[4] International Organization for Standardization. ISO 25178-6:2016 geometrical product specifications (GPS) - surface texture: Areal - part 6: Classification of methods for measuring surface texture, 2010-02. URL https://www.iso.org/standard/42896.html.

[5] International Organization for Standardization. ISO 251782:2012 geometrical product specifications (GPS) - surface texture: Areal - part 2: Terms, definitions and surface texture parameters, 2012-04. URL https://www.iso.org/ standard/42785.html.

[6] International Organization for Standardization. ISO 1328$1: 2013$ (en) cylindrical gears - ISO system of flank tolerance classification - part 1: Definitions and allowable values of deviations relevant to flanks of gear teeth, 2013-03. URL https://www.iso.org/obp/ui/\#iso:std:iso:1328:-1:ed-2:v1:en.

[7] International Organization for Standardization. ISO 1661071:2014 geometrical product specifications (GPS) - filtration - part 71: Robust areal filters: Gaussian regression filters, 2014-09. URL https://www.iso.org/standard/60159.html.

[8] International Organization for Standardization. ISO 251781:2016 geometrical product specifications (GPS) - surface texture: Areal - part 1: Indication of surface texture, 201604. URL https://www.iso.org/standard/46065.html.

[9] A. M. Müller and T. Hausotte. Comparison of different measures for the single point uncertainty in industrial x-ray computed tomography. In 9th Conference on Industrial Computed Tomography. e-Journal of Nondestructive Testing, 2019. URL http://www.ndt.net/iCT2019.

[10] A. M. Müller and T. Hausotte. Utilization of single point uncertainties for geometry element regression analysis in dimensional x-ray computed tomography. In 9th Conference on Industrial Computed Tomography. e-Journal of Nondestructive Testing, 2019. URL http://www.ndt.net/iCT2019.

[11] A. M. Müller, F. Wohlgemuth, and T. Hausotte. Simulationbased correction of systematic errors for ct measurements. In NDT.net 2018-02, 2018. URL http://www.ndt.net/article/ ctc2018/papers/ICT2018_paper_id127.pdf.

[12] A. M. Müller, T. Oberleiter, K. Willner, and T. Hausotte. Implementation of parameterized work piece deviations and measurement uncertainties into performant meta-models for an improved tolerance specification: accepted contribution. Proceedings of the International Conference on Engineering Design, ICED, 2019. 